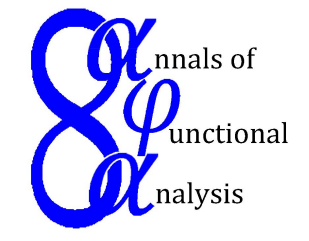

Ann. Funct. Anal. 5 (2014), no. 1, 51-55

$\mathscr{A}$ NNALS OF $\mathscr{F}$ UNCTIONAL $\mathscr{A}$ NALYSIS

ISSN: 2008-8752 (electronic)

URL:www.emis.de/journals/AFA/

\title{
SOME BANACH ALGEBRA PROPERTIES IN THE CARTESIAN PRODUCT OF BANACH ALGEBRAS
}

\author{
H. V. DEDANIA AND H. J. KANANI* \\ Dedicated to Professor T. Ando with respect \\ Communicated by M. S. Moslehian
}

\begin{abstract}
For semisimple, commutative Banach algebras $\mathcal{A}$ and $\mathcal{B}$, some Banach algebra properties of the Cartesin product $\mathcal{A} \times \mathcal{B}$ are characterized in terms of $\mathcal{A}$ and $\mathcal{B}$. A couple of results are also proved for non-commutative Banach algebras.
\end{abstract}

\section{INTRODUCTION}

Let $\left(\mathcal{A},\|\cdot\|_{\mathcal{A}}\right)$ and $\left(\mathcal{B},\|\cdot\|_{\mathcal{B}}\right)$ be semisimple, commutative Banach algebras. Let $\mathcal{A} \times \mathcal{B}$ denote their Cartesian product. Then $\mathcal{A} \times \mathcal{B}$ is a semisimple, commutative Banach algebra with co-ordinatewise product and with maximum norm; namely,

$$
\|(a, b)\|:=\max \left\{\|a\|_{\mathcal{A}},\|b\|_{\mathcal{B}}\right\} \quad(a \in \mathcal{A} ; b \in \mathcal{B}) .
$$

Note that the Gelfand space (resp., Shilov boundary) of $\mathcal{A} \times \mathcal{B}$ is homeomorphic to the topological sum of the Gelfand spaces (resp., Shilov boundaries) of $\mathcal{A}$ and $\mathcal{B}$. Based on this result, we prove that the properties like UUNP, QDZP, TAP, etc. are carried forward from $\mathcal{A}$ and $\mathcal{B}$ to $\mathcal{A} \times \mathcal{B}$ and vice-versa. Note that not all properties of $\mathcal{A}$ and $\mathcal{B}$ can be carried forward to $\mathcal{A} \times \mathcal{B}$. For example, let $\mathcal{A}=\mathcal{B}=\mathbb{C}$. Then they are division algebras but their cartesian product $\mathcal{A} \times \mathcal{B}=\mathbb{C}^{2}$ is not a division algebra.

Date: Received: 6 May 2013; Accepted: 11 June 2013.

* Corresponding author.

2010 Mathematics Subject Classification. Primary 46J05; Secondary 46K05.

Key words and phrases. Banach algebra, Cartesian product, UUNP, TAP, SEP.. 


\section{Main Results on $\mathcal{A} \times \mathcal{B}$}

To set notations, we start with the definition of sum topology [3, p-33].

Definition 2.1. Let $\left(X, \tau_{1}\right)$ and $\left(Y, \tau_{2}\right)$ be topological spaces such that $X$ and $Y$ are disjoint. Let $\tau=\left\{U \subset X \cup Y: U \cap X \in \tau_{1}\right.$ and $\left.U \cap Y \in \tau_{2}\right\}$. Then $\tau$ is a topology on $X \cup Y$, which is the sum topology on $X \cup Y$. The topological space $(X \cup Y, \tau)$ is the topological sum of $X$ and $Y$, which will be denoted by $X \biguplus Y$.

Let $\Delta(\mathcal{A})$ be the Gelfand space and $\partial \mathcal{A}$ be the Shilov boundary of $\mathcal{A}$. For $a \in \mathcal{A}$, let $\widehat{a}: \Delta(\mathcal{A}) \longrightarrow \mathbb{C}$ be defined as $\widehat{a}(\varphi)=\varphi(a)(\varphi \in \Delta(\mathcal{A}))$.

The following result is proved in [6] for $\mathcal{A}$ and $\mathcal{B}$ being unital. Because of the identities, the Gelfand spaces are compact Hausdorff spaces and the proof becomes much easier. Here we prove it without assuming identities and with different method.

Theorem 2.2. $\Delta(\mathcal{A} \times \mathcal{B}) \cong \Delta(\mathcal{A}) \biguplus \Delta(\mathcal{B})$ and $\partial(\mathcal{A} \times \mathcal{B}) \cong \partial \mathcal{A} \biguplus \partial \mathcal{B}$.

Proof. Note that $\mathcal{A} \times \mathcal{B}$ is the direct sum of the two closed ideals $\mathcal{A} \times\{0\}$ and $\{0\} \times \mathcal{B}$. So $\Delta(\mathcal{A} \times \mathcal{B})=\Delta(\mathcal{A}) \cup \Delta(\mathcal{B})$ set theoretically. By [4, Lemma 2.2.15], $\Delta(\mathcal{A})$ and $\Delta(\mathcal{B})$ are open in $\Delta(\mathcal{A} \times \mathcal{B})$ carrying the topologies induced from $\Delta(\mathcal{A} \times \mathcal{B})$. Thus $\Delta(\mathcal{A} \times \mathcal{B}) \cong \Delta(\mathcal{A}) \biguplus \Delta(\mathcal{B})$.

Now, let $\widehat{a} \in \widehat{\mathcal{A}}$. Since $\widehat{\mathcal{A}} \cup \widehat{\mathcal{B}}=\widehat{\mathcal{A} \times \mathcal{B}}$, we have $\widehat{a}=\widehat{(a, 0)}$. Since $\widehat{(a, 0)}$ assumes its maximum on $\partial(\mathcal{A} \times \mathcal{B})$, the $\widehat{a}$ would assume its maximum on $\partial(\mathcal{A} \times \mathcal{B})$. So $\partial(\mathcal{A} \times \mathcal{B})$ is a closed boundary for $\mathcal{A}$. Since $\partial \mathcal{A}$ is the smallest closed boundary for $\mathcal{A}$, we get $\partial \mathcal{A} \subset \partial(\mathcal{A} \times \mathcal{B})$. Similarly, $\partial \mathcal{B} \subset \partial(\mathcal{A} \times \mathcal{B})$. Hence $\partial \mathcal{A} \cup \partial \mathcal{B} \subset \partial(\mathcal{A} \times \mathcal{B})$. For the reverse inclusion, let $(a, b) \in \mathcal{A} \times \mathcal{B}$. Then $\widehat{(a, b)}=\widehat{a}$ or $\widehat{(a, b)}=\widehat{b}$. If $\widehat{(a, b)}=\widehat{a}$, then $\widehat{(a, b)}$ attains its maximum on $\partial \mathcal{A}$. If $\widehat{(a, b)}=\widehat{b}$, then $\widehat{(a, b)}$ attains its maximum on $\partial \mathcal{B}$. Hence $\partial \mathcal{A} \cup \partial \mathcal{B}$ is a closed boundary for $\mathcal{A} \times \mathcal{B}$. Since $\partial(\mathcal{A} \times \mathcal{B})$ is the smallest closed boundary for $\mathcal{A} \times \mathcal{B}$, we have $\partial(\mathcal{A} \times \mathcal{B}) \subset \partial \mathcal{A} \cup \partial \mathcal{B}$. Hence $\partial(\mathcal{A} \times \mathcal{B}) \cong \partial \mathcal{A} \biguplus \partial \mathcal{B}$.

Now we characterize some Banach algebra properties of $\mathcal{A} \times \mathcal{B}$ in terms of $\mathcal{A}$ and $\mathcal{B}$. We start with the unique uniform norm property (UUNP) which was introduced by Bhatt and Dedania [1].

Definition 2.3. A norm $\|\cdot\|$ (not necessarily complete) on $\mathcal{A}$ is a uniform norm if $\left\|a^{2}\right\|=\|a\|^{2}(a \in \mathcal{A})$. The Banach algebra $\mathcal{A}$ has unique uniform norm property $(U U N P)$ if it admits exactly one uniform norm.

Theorem 2.4. $\mathcal{A} \times \mathcal{B}$ has $U U N P$ if and only if $\mathcal{A}$ and $\mathcal{B}$ have $U U N P$.

Proof. Let $\mathcal{A} \times \mathcal{B}$ have UUNP. Let $F \subset \Delta(\mathcal{A})$ be a closed set of uniqueness for $\mathcal{A}$. Then by definition of sum topology $F \cup \Delta(\mathcal{B})$ is a closed subset of $\Delta(\mathcal{A}) \cup \Delta(\mathcal{B})=$ $\Delta(\mathcal{A} \times \mathcal{B})$. Moreover, it is also a set of uniqueness for $\mathcal{A} \times \mathcal{B}$. Since $\mathcal{A} \times \mathcal{B}$ has UUNP, by [1, Theorem 2.3], $\partial \mathcal{A} \cup \partial \mathcal{B}=\partial(A \times \mathcal{B}) \subset F \cup \Delta(\mathcal{B})$. Since $\Delta(\mathcal{A})$ and $\Delta(\mathcal{B})$ are disjoint, $\partial \mathcal{A} \subset F$. Thus $\partial \mathcal{A}$ is the smallest closed set of uniqueness for $\mathcal{A}$. Hence, by [1, Theorem 2.3], $\mathcal{A}$ has UUNP. Similarly, we can show that $\mathcal{B}$ has UUNP. 
Conversely, assume that $\mathcal{A}$ and $\mathcal{B}$ have UUNP. Let $F \subset \Delta(\mathcal{A} \times \mathcal{B})$ be a closed set of uniqueness for $\mathcal{A} \times \mathcal{B}$. Then $F=(F \cap \Delta(\mathcal{A})) \cup(F \cap \Delta(\mathcal{B}))$. Let $F_{\mathcal{A}}=$ $F \cap \Delta(\mathcal{A})$. Then $F_{\mathcal{A}}$ is a closed set of uniqueness for $\mathcal{A}$. Since $\mathcal{A}$ has UUNP, by [1, Theorem 2.3], $\partial \mathcal{A} \subset F_{\mathcal{A}}$. Similarly $\partial \mathcal{B} \subset F_{\mathcal{B}}$. Hence, by Theorem 2.2, $\partial(\mathcal{A} \times \mathcal{B})=\partial \mathcal{A} \cup \partial \mathcal{B} \subset F_{\mathcal{A}} \cup F_{\mathcal{B}}=F$. Thus $\partial(\mathcal{A} \times \mathcal{B})$ is the smallest closed boundary of $\mathcal{A} \times \mathcal{B}$. Hence, again by [1, Theorem 2.3], $\mathcal{A} \times \mathcal{B}$ has UUNP.

Definition 2.5. [4, p-198] $\mathcal{A}$ is regular if for every closed set $F \subset \Delta(\mathcal{A})$ and $\varphi \in \Delta(\mathcal{A}) \backslash F$, there exists $a \in \mathcal{A}$ such that $\widehat{a}(\varphi)=1$ and $\left.\widehat{a}\right|_{F}=0$.

The next result follows from [4, Theorem 4.3.8]. However, we prove it here with elementary arguments.

Theorem 2.6. $\mathcal{A} \times \mathcal{B}$ is regular iff $\mathcal{A}$ and $\mathcal{B}$ are regular.

Proof. Let $\mathcal{A} \times \mathcal{B}$ be regular. Let $F$ be a closed subsets of $\Delta(\mathcal{A})$ and let $\phi \in$ $\Delta(\mathcal{A}) \backslash F$. Then $F$ is closed in $\Delta(\mathcal{A} \times \mathcal{B})$ and $\varphi \in \Delta(\mathcal{A} \times \mathcal{B}) \backslash F$. By the hypothesis, there exists $(a, b) \in \mathcal{A} \times \mathcal{B}$ such that $\left.\widehat{(a, b)}\right|_{F}=0$ and $\widehat{(a, b)}(\varphi)=1$. But $\widehat{(a, b)}=\widehat{(a, 0)}=\widehat{a}$ on $\Delta(\mathcal{A})$. Hence $\left.\widehat{a}\right|_{F}=0$ and $\widehat{a}(\varphi)=1$. Thus $\mathcal{A}$ is regular. Similarly, $\mathcal{B}$ is regular.

Conversely, let $\mathcal{A}$ and $\mathcal{B}$ be regular. Let $F$ be a closed subsets of $\Delta(\mathcal{A} \times \mathcal{B})$ and $\varphi \in \Delta(\mathcal{A} \times \mathcal{B}) \backslash F$. Let $F_{\mathcal{A}}=F \cap \Delta(\mathcal{A})$ and $F_{\mathcal{B}}=F \cap \Delta(\mathcal{B})$. Then $F_{\mathcal{A}}$ and $F_{\mathcal{B}}$ are closed subsets of $\Delta(\mathcal{A})$ and $\Delta(\mathcal{B})$ respectively and $\varphi \in \Delta(\mathcal{A}) \backslash F_{\mathcal{A}}$ or $\varphi \in \Delta(\mathcal{B}) \backslash F_{\mathcal{B}}$. Suppose $\varphi \in \Delta(\mathcal{A}) \backslash F_{\mathcal{A}}$. Then, by the hypothesis, there exists $a \in \mathcal{A}$ such that $\left.\widehat{a}\right|_{F_{\mathcal{A}}}=0$ and $\widehat{a}(\varphi)=1$. Then $\left.\widehat{(a, 0)}\right|_{F}=\left.\widehat{a}\right|_{F_{\mathcal{A}}}=0$ and $\widehat{(a, 0)}(\varphi)=\widehat{a}(\varphi)=0$. Similarly, if $\varphi \in \Delta(\mathcal{B}) \backslash F_{\mathcal{B}}$, then there exists $b \in \mathcal{B}$ such that $\left.\widehat{b}\right|_{F_{\mathcal{B}}}=0$ and $\widehat{b}(\varphi)=1$. Then $\left.\widehat{(0, b)}\right|_{F}=\left.\widehat{b}\right|_{F_{\mathcal{B}}}=0$ and $\widehat{(0, b)}(\varphi)=\widehat{b}(\varphi)=0$. Hence $\mathcal{A} \times \mathcal{B}$ is regular.

Definition 2.7. [5, Definition 4, p-71] $\mathcal{A}$ has Quasi Divisior of Zero Property $(Q D Z P)$ if there exists an open set $G \subset \Delta(\mathcal{A})$ such that

(1) $\partial \mathcal{A} \subset \bar{G}$.

(2) For every open subset $U$ of $G$, there exists $a \in \mathcal{A}$ and a non-empty open subset $V$ of $U$ such that $\widehat{a}=1$ on $V$ and $\widehat{a}=0$ on $U^{c}$.

Theorem 2.8. $\mathcal{A} \times \mathcal{B}$ has $Q D Z P$ iff $\mathcal{A}$ and $\mathcal{B}$ have QDZP.

Proof. Let $\mathcal{A} \times \mathcal{B}$ have QDZP. Then there exists an open set $G \subset \Delta(\mathcal{A} \times \mathcal{B})$ which satisfies the following properties.

(1) $\partial(\mathcal{A} \times \mathcal{B}) \subset \bar{G}$.

(2) For each open subset $U$ of $G$, there exists $(a, b) \in \mathcal{A} \times \mathcal{B}$ and a nonempty open subset $V$ of $U$ such that $\widehat{(a, b)}=1$ on $V$ and $\widehat{(a, b)}=0$ on $U^{c}$.

Let $G_{\mathcal{A}}=G \cap \Delta(\mathcal{A})$. Then $G_{\mathcal{A}}$ will be open in $\Delta(\mathcal{A})$ and $\partial \mathcal{A} \subset \overline{G_{\mathcal{A}}}$. Now let $U \subset G_{\mathcal{A}}$ be open. Then $U$ will be open in $G$ also. Hence, by the hypothesis, there exist $(a, b) \in \mathcal{A} \times \mathcal{B}$ and an open set $\phi \neq V \subset U$ satisfying (2) above. Since $U \subset \Delta(\mathcal{A})$, we have $\widehat{a}=\widehat{(a, 0)}=\widehat{(a, b)}$. Hence $\mathcal{A}$ has QDZP. Similarly, $\mathcal{B}$ has QDZP. 
Conversely, suppose $\mathcal{A}$ and $\mathcal{B}$ have QDZP. Then there exist open subsets $G_{\mathcal{A}} \subset$ $\Delta(\mathcal{A})$ and $G_{\mathcal{B}} \subset \Delta(\mathcal{B})$ which satisfies the properties in the definition of QDZP. Let $G=G_{\mathcal{A}} \cup G_{\mathcal{B}}$. Then $\partial(\mathcal{A} \times \mathcal{B})=\partial \mathcal{A} \cup \partial \mathcal{B} \subset \bar{G}_{\mathcal{A}} \cup \bar{G}_{\mathcal{B}}=\overline{G_{\mathcal{A}} \cup G_{\mathcal{B}}}=\bar{G}$. Let $U \subset G$ be open. Then $U_{\mathcal{A}}=U \cap G_{\mathcal{A}}$ and $U_{\mathcal{B}}=U \cap G_{\mathcal{B}}$ are open in $G_{\mathcal{A}}$ and $G_{\mathcal{B}}$, respectively. Hence there exist $a \in \mathcal{A}$ and $b \in \mathcal{B}$ such that $\widehat{a}=0$ outside $U_{\mathcal{A}}, \widehat{a}=1$ on some open subset $\phi \neq V_{\mathcal{A}} \subset U_{\mathcal{A}}, \widehat{b}=0$ outside $U_{\mathcal{B}}$ and $\widehat{b}=1$ on some open subset $\phi \neq V_{\mathcal{B}} \subset U_{\mathcal{B}}$. Then $\widehat{(a, b)}=0$ outside $U=U_{\mathcal{A}} \cup U_{\mathcal{B}}$ and $\widehat{(a, b)}=1$ on $\phi \neq V_{\mathcal{A}} \cup V_{\mathcal{B}} \subset U$. Thus $\mathcal{A} \times \mathcal{B}$ has QDZP.

Definition 2.9. [5, Definition 5, p-72] Let $M \subseteq \mathcal{A}$ be a maximal ideal. A separating net for $M$ is a net $\left\{q_{\alpha}\right\}_{\alpha \in \Lambda}$ in $\mathcal{A}$ such that

(1) $\sup _{\alpha \in \Lambda} r_{\mathcal{A}}\left(q_{\alpha}\right)<\infty$;

(2) $\lim _{\alpha \rightarrow \infty} r_{\mathcal{A}}\left(a q_{\alpha}\right)=0(a \in M)$;

(3) There exists $b \in \mathcal{A}$ such that $q_{\alpha} b=q_{\alpha}(\alpha \in \Lambda)$;

(4) For each $\alpha \in \Lambda$, there exists $p_{\alpha} \in \mathcal{A}$ such that $p_{\alpha}+q_{\alpha}-p_{\alpha} q_{\alpha}=0$.

Definition 2.10. [5, Definition 5, p-72] $\mathcal{A}$ has Topological Annihilator Property $(T A P)$ if there exists a dense subset $D \subset \partial \mathcal{A}$ such that $\operatorname{ker} \varphi$ admits a separating net for every $\varphi \in D$.

Theorem 2.11. $\mathcal{A} \times \mathcal{B}$ has TAP iff $\mathcal{A}$ and $\mathcal{B}$ have TAP.

Proof. Let $\mathcal{A} \times \mathcal{B}$ have TAP. Then there exists dense subset $D \subset \partial(\mathcal{A} \times \mathcal{B})$ such that $\operatorname{ker} \varphi(\varphi \in D)$ admits a separating net. Let $D_{\mathcal{A}}=D \cap \partial \mathcal{A}$. Then $D_{\mathcal{A}}$ will be a dense subset of $\partial \mathcal{A}$. Let $\varphi_{\mathcal{A}} \in D_{\mathcal{A}}$. Define $\varphi(a, b)=\varphi_{\mathcal{A}}(a)((a, b) \in \mathcal{A} \times \mathcal{B})$. Then $\varphi \in D$. Hence $\operatorname{ker} \varphi$ admits a separating net say $\left(a_{\alpha}, b_{\alpha}\right)$. Then $\left(a_{\alpha}\right)$ will be a separating net for $\operatorname{ker} \varphi_{\mathcal{A}}$. Thus $\mathcal{A}$ has TAP. Similarly, $\mathcal{B}$ has TAP.

Conversely, let $\mathcal{A}$ and $\mathcal{B}$ have TAP. Then there exist dense subsets $D_{\mathcal{A}} \subset \partial \mathcal{A}$ and $D_{\mathcal{B}} \subset \partial \mathcal{B}$ such that each $\operatorname{ker} \varphi\left(\varphi \in D_{\mathcal{A}} \cup D_{\mathcal{B}}\right)$ admits a separating net. Then $D=D_{\mathcal{A}} \cup D_{\mathcal{B}}$ is a dense subset of $\partial \mathcal{A} \cup \partial \mathcal{B}=\partial(\mathcal{A} \times \mathcal{B})$. Hence $\mathcal{A} \times \mathcal{B}$ has TAP.

Note: For the rest, $\mathcal{A}$ and $\mathcal{B}$ are not assumed to be commutative.

Definition 2.12. [4, p-223] A norm $|\cdot|$ on $\mathcal{A}$ is spectral if $r_{\mathcal{A}}(a) \leq|a|(a \in \mathcal{A})$, where $r_{\mathcal{A}}(\cdot)$ is the spectral radious on $\mathcal{A}$. The algebra $\mathcal{A}$ has Spectral Extension Property (SEP) if every norm on $\mathcal{A}$ is spectral.

The SEP is a very important property in Banach algebras [4, p-222]). We do not know the converse of the following result even for commutative case.

Theorem 2.13. If $\mathcal{A} \times \mathcal{B}$ has $S E P$, then $\mathcal{A}$ and $\mathcal{B}$ have $S E P$.

Proof. Let $|\cdot|$ be a norm on $\mathcal{A}$. Define $|(a, b)|_{1}=|a|+\|b\|_{\mathcal{B}}$, where $\|\cdot\|_{\mathcal{B}}$ is the Banach algebra norm on $\mathcal{B}$. Since $\mathcal{A} \times \mathcal{B}$ has SEP, we have

$$
r_{\mathcal{A}}(a)=r_{\mathcal{A} \times \mathcal{B}}(a, 0) \leq|(a, 0)|_{1}=|a| \quad(a \in \mathcal{A}) .
$$

Thus $|\cdot|$ is spectral on $\mathcal{A}$, and so $\mathcal{A}$ has SEP. Similarly, $\mathcal{B}$ has SEP.

Definition 2.14. A norm $|\cdot|$ on $\mathcal{A}$ is semisimple if the completion of $(\mathcal{A},|\cdot|)$ is semisimple. The $\mathcal{A}$ has Weak Spectral Extension Property (WSEP) if every semisimple norm on $\mathcal{A}$ is spectral. 
If $\mathcal{A}$ is commutative, then $\mathcal{A}$ has UUNP iff $\mathcal{A}$ has WSEP [1, Proposition-2.1]. Thus the WSEP is a non-commutative analogue of the UUNP. Unfortunately, we do not know the converse of the following result also.

Theorem 2.15. If $\mathcal{A} \times \mathcal{B}$ has WSEP, then $\mathcal{A}$ and $\mathcal{B}$ have WSEP.

Proof. Let $|\cdot|_{\mathcal{A}}$ and $|\cdot|_{\mathcal{B}}$ be semisimple norms on $\mathcal{A}$ and $\mathcal{B}$, respectively. Define

$$
|(a, b)|=|a|_{\mathcal{A}}+|b|_{\mathcal{B}} \quad((a, b) \in \mathcal{A} \times \mathcal{B}) .
$$

Then $|\cdot|$ is a norm on $\mathcal{A} \times \mathcal{B}$. Note that $\left(\mathcal{A},|\cdot|_{\mathcal{A}}\right) \times\left(\mathcal{B},|\cdot|_{\mathcal{B}}\right) \cong(\mathcal{A} \times \mathcal{B},|\cdot|)$, where $(X,\||\cdot|\|)$ denotes the completion of $(X,\||\cdot|\|)$. Since $|\cdot|_{\mathcal{A}}$ and $|\cdot|_{\mathcal{B}}$ are semisimple norms on $\mathcal{A}$ and $\mathcal{B},\left(\mathcal{A},|\cdot|_{\mathcal{A}}\right) \times\left(\mathcal{B},|\cdot|_{\mathcal{B}}\right) \cong(\mathcal{A} \times \mathcal{B},|\cdot|)$ is semisimple. So, by the hypothesis, $|\cdot|$ is a spectral norm on $\mathcal{A} \times \mathcal{B}$. Hence,

$$
|a|_{\mathcal{A}}=|(a, 0)| \leq r_{\mathcal{A} \times \mathcal{B}}(a, 0)=r_{A}(a) .
$$

Thus $|\cdot|_{\mathcal{A}}$ is a spectral norm on $\mathcal{A}$. Similarly, $|\cdot|_{\mathcal{B}}$ is spectral on $\mathcal{B}$.

Acknowledgement. The work has been supported by UGC-SAP-DRS-II Grant No.F.510/3/DRS/2009 provided to the Department of Mathematics, Sardar Patel University, Vallabh Vidyanagar. The authors are very much thankful to the referee for fruitful suggestions in the manuscript.

\section{REFERENCES}

1. S.J. Bhatt and H.V. Dedania, Banach algebras with unique uniform norm, Proc. Amer. Math. Soc. 124 (1996), no. 2, 579-584.

2. F. Bonsall and J. Duncan, Complete Normed Algebras, Springer, Berlin, 1973.

3. N. Bourbaki, General Topology: Elements of Mathematics, Springer, Berlin, 1989.

4. E. Kaniuth, A Course in Commutative Banach Algebras, Springer, New York, 2009.

5. M.J. Meyer, Submultiplicative norms on Banach algebras, Ph.D. thesis, University of Oregon, 1989.

6. N.H. Shah, Cartesian Product of Banach algebras, M.Phil. Dissertation, Sardar Patel University, 2007.

${ }^{1}$ Department of Mathematics, Sardar Patel University, V. V. Nagar - 388 120, Gujarat, India.

E-mail address: hvdedania@yahoo.com

E-mail address: hitenmaths69@gmail.com 